\title{
Less Trust, Moore Verification: Determining the Accuracy of Third-Party Data through an Innovative Use of Attention Checks
}

Nathan Seltzer*

University of Wisconsin-Madison

\begin{abstract}
Sociologists increasingly rely on third-party internet panel platforms to acquire respondents and administer questionnaires. Yet, researchers have demonstrated that even samples sourced from well-respected and widely-adopted internet platforms such as Amazon's Mechanical Turk are often unable to screen out respondents who do not meet selection criteria requested by researchers. Here, I argue that researchers should proactively verify that third-party survey data is accurately sampled before considering it for analysis. I propose using survey "attention checks" as a methodological solution for researchers to determine whether data vendors have provided low quality data. In this short research note, I illustrate the approach by analyzing data from a consequential political opinion poll administered on behalf of an academic polling center by a third-party internet panel vendor for a special election in 2017. By assessing valid/invalid response choices of two overlapping geographic variables, I identify irregularities in the dataset that suggest that the sample included respondents who were not within the researchers' intended sampling frame. Attention checks provide a straightforward, inexpensive tool to improve the validity of research produced with internet-drawn samples.
\end{abstract}

Replication code: https://github.com/nathanseltzer/ltmv

*(Email: nseltzer@wisc.edu) University of Wisconsin, Madison. 8128 William H. Sewell Social Sciences Building, 1180 Observatory Drive, Madison Wisconsin, 53706. The author is grateful for feedback from Jenna Nobles, Myra Marx Ferree, Nora Cate Schaeffer, and Jessica West. This research was supported by a core grant to the Center for Demography and Ecology at UWMadison [P2C HD047873] as well as support from a training grant awarded to the Center for Demography and Ecology [T32 HD07014]. All errors are the author's own. 


\section{Less Trust, Moore Verification: Determining the Accuracy of Third-Party Data through an Innovative Use of Attention Checks}

Nathan Seltzer, University of Wisconsin-Madison

nseltzer@wisc.edu

Data collection for sociological research has changed in several fundamental ways over the past two decades. Declining response rates, reductions in federal funding, and rising survey costs have altered the context in which data for sociological research is produced (Massey and Tourangeau 2013; National Research Council 2013). At the same time, social scientists have embraced new modes of data collection, including internet-based survey designs (Groves 2011). The use of internet-based survey designs often entails contracting out sampling and survey administering to third-party data vendors - frequently for-profit marketing or polling firms - that draw respondents from ongoing internet-based panels (Hays, Liu, and Kapteyn 2015). This sort of third-party internet data has now been used in studies published in every major journal in the discipline (e.g. Edgell et al. 2016; Flores 2018; Hahl, Kim, and Zuckerman 2018; Kuwabara 2015; Pedulla and Thébaud 2015).

In light of this shift, I raise a straightforward but easily overlooked concern: samples from thirdparty internet panel vendors do not necessarily represent the target population requested by the researcher, even if sourced from well-respected and widely-adopted internet panel platforms. For instance, recent reports about systematic data quality issues from Amazon's Mechanical Turk platform demonstrate how a non-trivial number of respondents use location-masking methods to bypass geographic sampling frame criteria requested by researchers (Ahler, Roush, and Sood 2019; Dennis, Goodson, and Pearson 2018; Kennedy et al. 2018).

In this research note, I argue that researchers should proactively verify that third-party survey data is accurately sampled before considering it for analysis. Researchers often fail to do this accuracy check because they expect that third-party data vendors have pre-screened the sample based on their requested sampling frame criteria. Under the expectation that the provided sample has been accurately pre-screened, researchers might omit items from questionnaires that could otherwise be used to verify whether the sample matched the requested sampling frame. Unless screening items are explicitly included in the questionnaire, there is no way to validate whether 
respondents match the selection criteria. Importantly, researchers need to take steps to avoid the use of survey data which yield erroneous conclusions.

To illustrate the gravity of this concern, I present findings from a simple analysis of data from a political opinion poll administered on behalf of an academic-based polling center by a third-party internet panel vendor. This poll received sustained national press at the time of publication because of its startling findings and the substantial national attention placed on the electoral contest for which it measured public opinion. By assessing valid/invalid response choices to overlapping geographic variables, I identify irregularities in the dataset that suggest that the sample included respondents who were not within the researchers' intended sampling frame. As a means of proactively verifying the accuracy of survey data provided by third-party data vendors, I propose an innovative use of survey "attention checks."

\section{ATTENTION CHECKS FOR DATA VENDORS}

Attention checks are widely used by survey researchers to assess data quality by testing whether respondents have provided sufficient attention to survey instructions while answering questionnaires. These attention checks usually take the form of "trap" questions that should be correctly answered if the respondent has been cognitively engaged with the instructions of the survey, but incorrectly answered if the respondent is not paying attention or exerting minimal effort to complete the survey (Anduiza and Galais 2017).

While the conventional use of attention checks attempts to ascertain valid item responses by survey respondents, I propose that researchers use attention checks to verify whether data vendors have provided samples that match the sampling frame criteria requested by the researcher. I define data vendor attention checks as demographic questionnaire items with response categories that extend beyond the range of the intended sampling frame's demographic characteristics. Since researchers have a priori expectations of who should be in their sample, the distribution of responses to data vendor attention check questionnaire items should correspond to their expectations. Any substantive divergence in the distribution of demographic variables used to define the sampling frame would indicate that the sample includes respondents who were not 
intended to be in the sampling frame. If this is the case, then the data vendor has not paid sufficient attention to providing an accurate sample to the researcher.

In the example that follows, I illustrate how researchers can repurpose pre-existing questionnaire items and questionnaire paradata as ad-hoc attention checks to verify the accuracy of a sample provided by a third-party internet panel vendor.

\section{AN EXAMPLE: POLLING THE 2017 SPECIAL ALABAMA SENATE RACE}

Emerson College Polling, an academic-affiliated polling center at Emerson College, fielded a political survey in November 2017 that gauged support for senate candidates Roy Moore (Republican) and Doug Jones (Democrat) by registered and likely Alabama voters in the special Alabama senate race which was to be held on December $12^{\text {th }}, 2017$. The findings of the poll received substantial state and national press given the timing of its release, which was conducted in the days following the Washington Post publishing an article that detailed a series of sexual abuse allegations against Roy Moore. Accordingly, the Emerson poll provided the first scientific survey of Alabama voters' candidate preferences in the wake of this major campaign shakeup. The results of the poll suggested that despite the allegations, Roy Moore held $55 \%$ of the vote while Doug Jones held $45 \%$ of the vote in a two-candidate race (margin of error $+/-3.9 \%$ ).

Emerson College Polling is a charter member of the American Association for Public Opinion Research's Transparency Initiative (AAPOR TI) which encourages the full disclosure of data and methodology of public opinion surveys to the public as a means of transparency and replication. Emerson College Polling releases raw datasets and disclosure forms for all their publiclyreleased surveys. As noted on their website, “These resources will be publicly available to students, teachers, researchers, and practitioners. As an academic institution focused on advancing the understanding of public opinion research, we invite all these groups to use, study and critique our methods and results in any way they wish and to share their findings with us in a collaborative manner" (Emerson College Polling 2017b).

In this analysis, I examine the Emerson College Poll from November 13, 2017. According to the press release and the methodology disclosure form (Emerson College Polling 2017a), the sample was acquired through two modes of administration: interactive voice response (IVR) of landline 
numbers with a voter phone number file supplied by Aristotle, LLC, and an online panel survey supplied by Opinion Access Corp., LLC. The stated sampling frame consisted of registered and likely voters in the state of Alabama. In the publicly released dataset, the IVR sample consisted of 628 respondents and the internet panel consisted of 324 respondents. ${ }^{1}$

\section{ATTENTION CHECKS THROUGH A PRIORI EXPECTATIONS OF VARIABLE DISTRIBUTIONS}

To verify whether the internet sample of the November 13, 2017 Emerson College Poll was comprised of valid Alabama respondents, I examine the joint frequency distribution of two overlapping geographic variables in the dataset: county of residence ("county") and US congressional district ("USC District”). In the internet sample, respondents were asked to, first, indicate their county of residence from a list of all 67 Alabama counties and, second, identify their congressional district from a map that displayed all 7 Alabama congressional districts (Figure 1). The IVR landline phone sample dataset includes variables for county and congressional district, although the publicly-released methodology and dataset leave unclear how these variables were acquired in this mode; however, it is likely that the voter landline phone file acquired through Aristotle, LLC included these attributes.

Examining the univariate frequency distributions of these variables would not sufficiently verify the accuracy of the sampling frame because all response categories indicate a geographic location within Alabama. Moreover, there was no variable in the internet sample questionnaire that asked respondents about their state of residence. However, examining the joint frequency distribution of both the county and congressional district variables reveals whether respondents indicated illogical county-district pairs.

Alabama counties are mostly nested within congressional districts, although there are several counties that overlap with two or three congressional districts (Figure 1). As a result, we should expect that congressional districts are non-randomly distributed within counties. The a priori

\footnotetext{
${ }^{1}$ Although the dataset has 628 IVR observations, the press release and disclosure form state that only 317 IVR observations were used to estimate the polling results. For the purposes here, I use all 628 IVR observations in the raw dataset. The purpose of this analysis is not to replicate the findings of the poll, but to verify the accuracy of the sample.
} 
expectation of the joint distribution would be that most counties should only have respondents in a single congressional district. Additionally, we should expect that respondents correctly matched their county and corresponding congressional district - there should be no ambiguity, with exception of the possibility of minimal respondent error.

In Figure 2, I graph the joint frequency distribution of respondents by their counties and congressional districts for both the internet sample $(\mathrm{N}=628)$ and the IVR phone sample $(\mathrm{N}=324)$. The rows of the graph correspond to county of residence while the columns correspond to the respondents' specified congressional districts. The dark blue boxes represent clusters of one or more respondents, while the light grey boxes represent no respondents. Importantly, the red xmarks indicates valid responses; all other cells in the heat map represent illogical and invalid county-district pairs.

In this figure, the IVR sample matches our a priori expectation for how congressional districts should be distributed within counties - most counties only have respondents in one congressional district. Moreover, all respondents in the IVR sample have correctly matched county-district pairs. $^{2}$

For the internet sample, however, there are a substantial number of respondents who incorrectly matched their county and congressional district. 117 out of the 324 respondents $(36.1 \%)$ were unable to accurately match their county of residence to their US congressional district when presented with a map of Alabama which displayed both. Or stated differently, $36.1 \%$ of respondents were unable to locate their county of residence on a map. In Autauga county, for instance, which is in central Alabama and entirely within District \#2, none of the respondents from the internet sample selected District \#2. Instead, they indicated that their congressional district was either District \#1, District \#3, District \#4, or District \#7, all of which are illogical county-district pairs.

It is unclear why respondents in the internet sample failed to correctly match their congressional districts to their county of residences. In the online questionnaire, respondents were provided a map that had congressional districts transposed over county boundaries (i.e. Figure 1) and were

\footnotetext{
${ }^{2}$ It is likely that the voter phone number file supplied by Aristotle, LLC already included this information since it is based on voter records. Nevertheless, the IVR sample demonstrates what the correct joint distribution should look like.
} 
then asked to indicate their congressional district. This should have been a simple classification task for respondents if they had knowledge of where they lived within their state of residency.

One plausible explanation for this discrepancy is that respondents rushed through the online questionnaire and engaged in satisficing (Krosnick, Narayan, and Smith 1996). To evaluate evidence of satisficing, Figure 3 displays the relative frequency distribution of county of residence, arranged alphabetically, for (a) the internet sample, (b) the IVR phone sample, and (c) the 2017 population of Alabama estimated by the U.S. Census Bureau. This figure demonstrates that the internet sample had a higher frequency of respondents reporting residence in counties at the beginning of the alphabet than would be expected by the 2017 population. In contrast, the distribution of responses for the IVR sample approximately matches the 2017 population. If the internet sample distribution is an indication of satisficing, it still calls into question the validity of the responses.

However, another explanation for the cluster of responses at the beginning of the alphabet is that respondents who did not reside in Alabama were forced to choose a response to the county of residence item in order to complete the questionnaire. These out-of-state respondents might have attempted to select their actual county of residence but were only able to do so if the name of their county of residence corresponded to the name of an Alabama county. For instance, about $5 \%$ of the internet sample indicated that they lived in Washington county - arguably the most common county name in the U.S. However, the actual population residing Washington county, Alabama in 2017 was estimated to comprise just $0.34 \%$ of the total state population $(16,531$ residents). If this account explains the irregular distribution of county of residence in the internet sample, it emphasizes how respondents were both (a) improperly pre-screened for state of residence by the data vendor, and (b) never explicitly asked about state of residence in the questionnaire.

\section{ROBUSTNESS ANALYSIS: IP ADDRESS GEOLOCATION}

The publicly-accessible dataset for the November 13, 2017 poll included respondent IP addresses for the internet sample which were logged during data collection. Given the irregularities uncovered through the prior analysis, I used IP address geolocation as an additional means of verifying whether respondents were located within the state of Alabama (Ahler et al. 2019). I used an R package, IPtoCountry (Robertson et al. 2016), to geolocate respondent IP addresses at 
the state-level using a downloaded, open source database of IP integer ranges. I further validated these results using an additional geolocation R Package, ipapi (Rudis 2015).

Out of the 324 respondents in the internet sample, 59 were geolocated to Alabama based on the IPtoCountry package and 83 were geolocated to Alabama based on the ipapi package. ${ }^{3}$ In contrast, 89 (IPtoCountry) and 128 (apipi) respondents were geolocated to Virginia.

Accordingly, the findings of this robustness analysis would suggest that only $18.2 \%$ to $25.6 \%$ of the respondents in the internet panel sample completed the survey using IP addresses registered to Alabama. Any statistical inference used on this sample to measure political opinions of Alabama voters would then be likely be highly invalid.

Figure 4 graphs the joint frequency distribution of respondents by county of residence and congressional district for the Alabama IP Address subsample and the non-Alabama IP address subsample. The results show that respondents with geolocated Alabama IP addresses have counties of residence that mostly correspond to the correct congressional district. For those whose IP addresses were geolocated to outside Alabama, the correspondence of counties to congressional districts includes a mixture of valid and invalid county-district pairs. Overall, the findings presented here further increase the possibility that a sizeable portion of the respondents from the internet sample were not within the intended sampling frame.

\section{DISCUSSION/CONCLUSION}

Third-party internet panel vendors provide a cost-effective and time-efficient option for conducting survey research, especially for researchers with few resources. The findings presented here emphasize the importance of verifying the quality and accuracy of survey data from internet panel vendors before disseminating the findings to a broader audience. Ultimately, it is the researcher's responsibility to determine the fidelity of the data they use in their analysis. This analysis demonstrates the utility of using data vendor attention checks. Data vendors have financial aims and motives that do not necessarily align with the quality standards expected of rigorous academic research, and therefore researchers should by default be skeptical of the accuracy of third-party data. Besides asking data vendors to provide their methodology,

\footnotetext{
${ }^{3}$ It is possible that other IP address databases might yield different estimates.
} 
researchers must take it upon themselves to create accuracy checks which can be used to determine whether the data vendor properly administered the survey. 


\section{REFERENCES}

Ahler, Douglas J., Carolyn E. Roush, and Gaurav Sood. 2019. The Micro-Task Market for "Lemons": Collecting Data on Amazon's Mechanical Turk.

Anduiza, Eva and Carol Galais. 2017. "Answering Without Reading: IMCs and Strong Satisficing in Online Surveys." International Journal of Public Opinion Research 29(3):497-519.

Dennis, Sean A., Brian M. Goodson, and Chris Pearson. 2018. Electronic Copy Available at: Https://Ssrn.Com/Abstract=3233954.

Edgell, Penny, Douglas Hartmann, Evan Stewart, and Joseph Gerteis. 2016. "Atheists and Other Cultural Outsiders: Moral Boundaries and the Non-Religious in the United States.” Social Forces 95(2):607-38.

Emerson College Polling. 2017a. "November 13, 2017 Alabama Senate Poll [Data File, Press Release, and Disclosure Form]."

Emerson College Polling. 2017b. "Polling Society: Alumni \& Student Research." Retrieved (http://www.emerson.edu/communication-studies/emerson-college-polling-society/alumnistudent-research).

Flores, René D. 2018. “Can Elites Shape Public Attitudes Toward Immigrants?: Evidence from the 2016 US Presidential Election.” Social Forces 96(4):1649-90.

Groves, Robert M. 2011. “Three Eras of Survey Research.” Public Opinion Quarterly 75(5):861-71.

Hahl, Oliver, Minjae Kim, and Ezra W. Zuckerman. 2018. "The Authentic Appeal of the Lying Demagogue : Proclaiming the Deeper Truth about Political Illegitimacy." American Sociological Review 83(1):1-33.

Hays, Ron D., Honghu Liu, and Arie Kapteyn. 2015. "Use of Internet Panels to Conduct Surveys." 47(3):685-90.

Kennedy, Ryan, Scott Clifford, Tyler Burleigh, Ryan Jewell, and Philip Waggoner. 2018. The Shape of and Solutions to the MTurk Quality Crisis. 
Krosnick, Jon A., Sowmya Narayan, and Wendy R. Smith. 1996. "Satisficing in Surveys: Initial Evidence." New Directions for Evaluation 1996(70):29-44.

Kuwabara, Ko. 2015. “Do Reputation Systems Undermine Trust? Divergent Effects of Enforcement Type on Generalized Trust and Trustworthiness." American Journal of Sociology 120(5):1390-1428.

Massey, Douglas S. and Roger Tourangeau. 2013. "Where Do We Go From Here? Nonresponse and Social Measurement." The ANNALS of the American Academy of Political and Social Science (January):222-36.

National Research Council. 2013. "The Growing Problem of Nonresponse.” Pp. 7-39 in Nonresponse in Social Science Surveys: A Research Agenda. Washington, D.C.: The National Academies Press.

Pedulla, David S. and Sarah Thébaud. 2015. "Can We Finish the Revolution? Gender, WorkFamily Ideals, and Institutional Constraint.” American Sociological Review 80(1):116 -139.

Robertson, Ronald E., Felix W. Tran, Jonathan Mejia, and Christine Mourani. 2016. "IPtoCountry: Convert IP Addresses to Country Names or Full Location with Geoplotting. R Package Version 0.0.1.”

Rudis, Bob. 2015. "Ipapi: Geolocate IPv4/6 Addresses and/or Domain Names Using IpApi.Com's API. R Package Version 0.1." 
Figure 1. Alabama County and Congressional District Boundaries

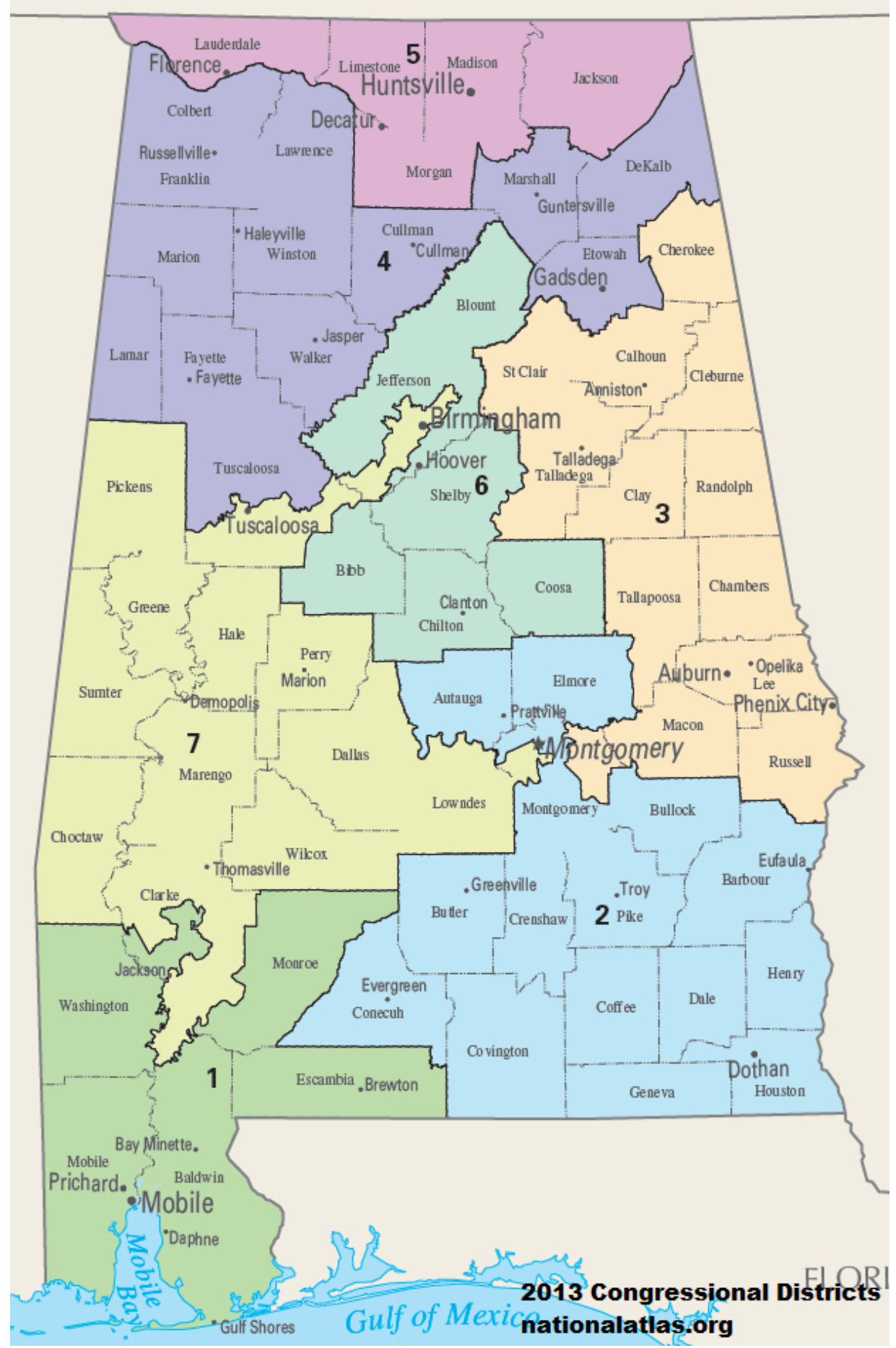

Source: Department of the Interior. (2014). National Atlas of the United States. "Map of Congressional Districts in the state of Alabama, reflecting district boundaries current to the 113th United States Congress." Retrieved on December 1, 2017 from "https://commons.wikimedia.org/wiki/File:Alabama_Congressional_Districts,_113th_Congress.t if" 
Figure 2. Binary Heat Map Depicting Joint Distribution of Counties of Residence and Congressional Districts for Respondents in the Internet and IVR Samples.

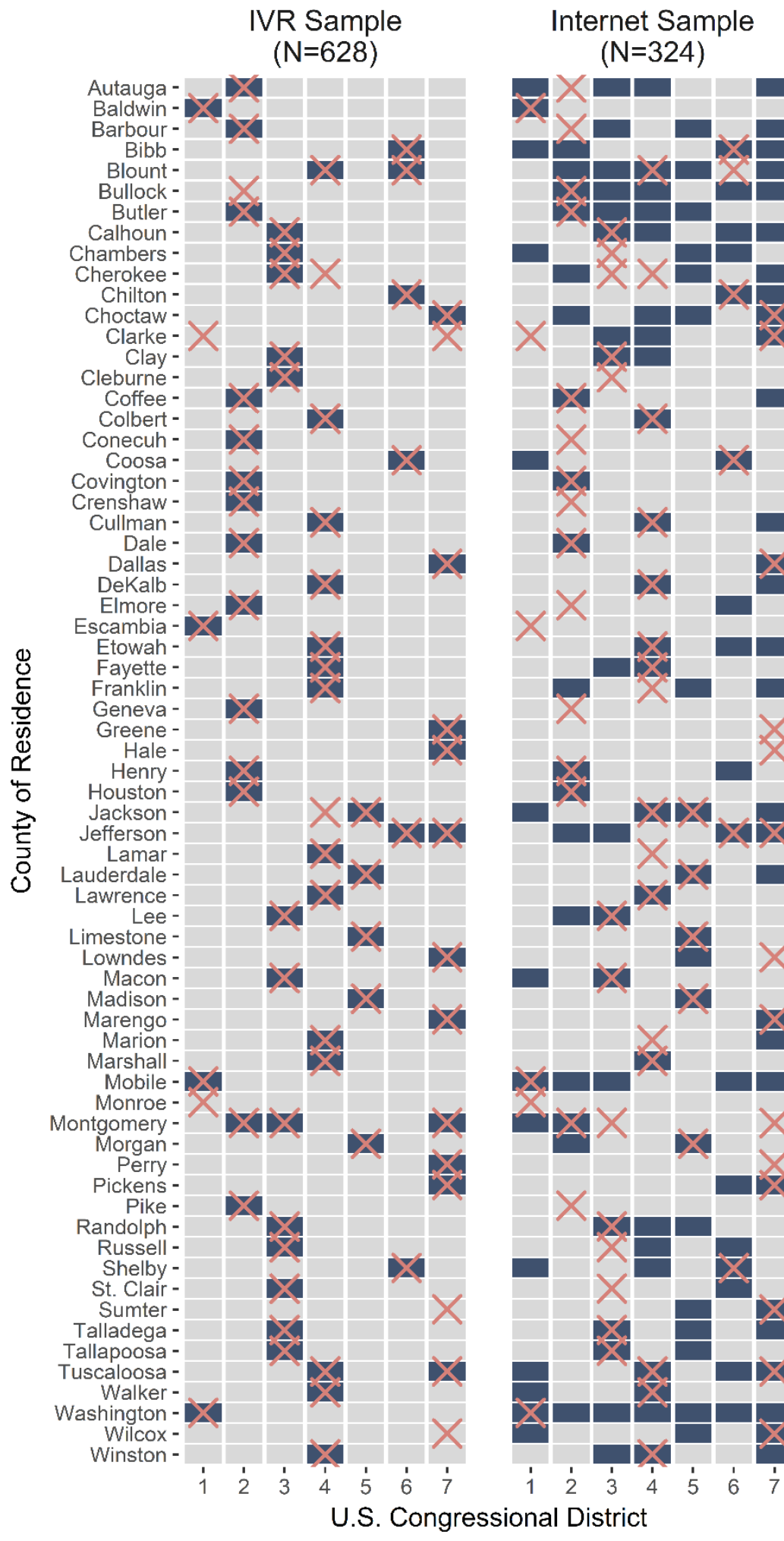

Actual Match

$X$ Yes

Any Respondents?

No

Yes 
Notes: Correct Match refers to valid/logical matches for counties and congressional districts. All other cells represent invalid/illogical county-district pairs. Blue cells refer to whether one or more respondents indicated that they lived in the corresponding county and congressional district. 
Figure 3. Relative Frequency Distributions of County of Residence for 2017 Population of Alabama, Internet Sample, and IVR Phone Sample.

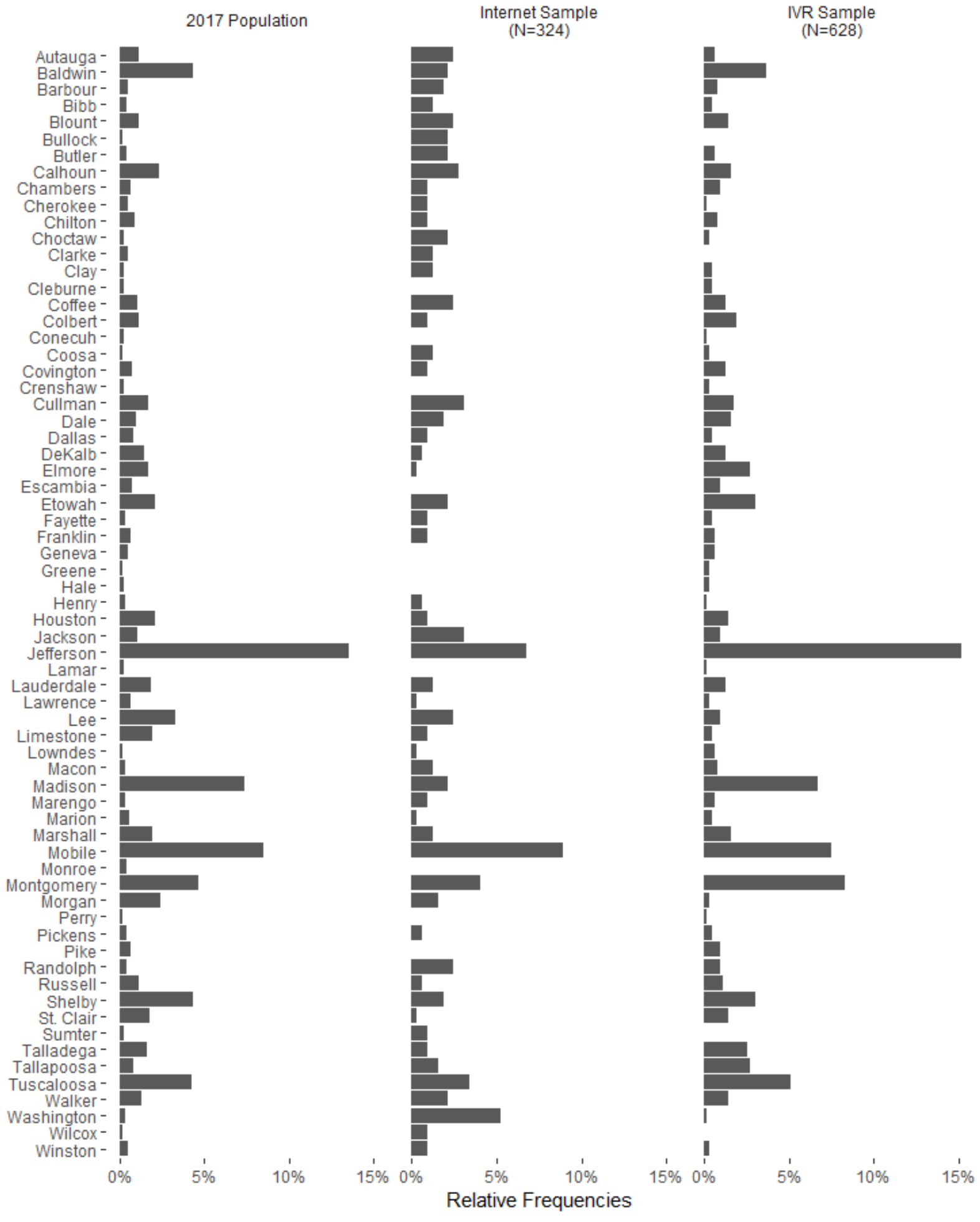


Figure 4. Binary Heat Map Depicting Joint Distribution of Counties of Residence and Congressional Districts for Respondents with Alabama and non-Alabama IP Addresses.



Actual Match

$X$ Yes

Any Respondents?

No
Yes 
Notes: Correct Match refers to valid/logical matches for counties and congressional districts. All other cells represent invalid/illogical county-district pairs. Blue cells refer to whether one or more respondents indicated that they lived in the corresponding county and congressional district. IP address geolocation conducted on downloaded, open-source database of IP integer ranges using the IPtoCountry R package (Robertson et al. 2016). 